\title{
Synthesis and Characterization of Bismuth (III) Iodomolybdate Inorganic Cation Exchanger
}

\author{
Subhash Chand, Seema, Rekha Rani, Vijayata \& Brajesh Pal \\ Chemistry Department Meerut College,Meerut (U.P. ) 250001 (India)
}

\begin{abstract}
Bismuth(III) Iodomolybdate, a new inorganic cation exchanger, was synthesized. The ion exchange capacity was determined by column method and $P^{H}$ titration method. The ion exchange capacity of the exchanger was found to be $1.2 \mathrm{meq} / \mathrm{g}$. The material has also been characterized on the basis of thermal stability, chemical stability, thermogravimetric analysis, $x$-ray diffraction pattern and infrared studies. Thermal stability shows ion exchange capacity of bismuth(III) Iodomolybdate decreases with increase in temperature and it also revealed that weight of bismuth(III) Iodomolybdate decrease with increase in temperature. Chemical resistivity of the material was assessed in various acidic and basic media. Distribution studies find the synthesized ion exchanger selective for $\mathrm{Ni}^{++}$ions.
\end{abstract}

Key words: Bismuth(III) Iodomolybdate, Inorganic cation exchanger, Ion exchange capacity, Thermal stability, Chemical stability and Distribution studies.

\section{Introduction}

In separation science two or more components are separated by various techniques, eg. ion exchange, solvent extraction etc. Synthesis, Characterization and ion exchange properties of different types of ion exchangers based on different metal ions such as $\mathrm{Zr}(\mathrm{IV}), \mathrm{Ce}(\mathrm{IV}), \mathrm{Sr}(\mathrm{IV}), \mathrm{Th}(\mathrm{V}), \mathrm{Sb}(\mathrm{V}), \mathrm{Sb}(\mathrm{III}), \mathrm{Bi}(\mathrm{III})$ etc. have been reported ${ }^{14-35}$. The exchangers have wide range of properties, selectivity and reproducibility in their behaviour. Recently most literature have been concentrated on ion exchangers based on zirconium(IV), antimony(III), titanium, and tin (IV) and only a few studies of the exchangers have been reported based on bismuth(III) an element of 15 th group in the periodic table.

\section{Experimental}

Reagents and chemicals - Bismuth nitrate, potassium iodate and sodium molybdate were obtained from C.D.H. Ltd. (India). All the chemicals such as, sodium chloride, sodium hydroxide, sodium salt of EDTA, hexamine, hydroxyl ammonium chloride, triethanolamine, erichrome black-T , etc. of AR grade were used.

\section{Apparatus :-}

$\mathrm{P}^{\mathrm{H}}$ measurements were done using a Toshniwal research $\mathrm{P}^{\mathrm{H}}$ meter model $\mathrm{P}^{\mathrm{H}} 110 . \mathrm{TGA}, \mathrm{X}-\mathrm{RD} \& \mathrm{IR}$ of the exchanger were obtained from IIT, Roorkee. Perkin Elmer (Pyris Diamond) in alumina pan with a current of nitrogen for TGA, Thermo nicolet IR spectrophotometer for IR and Philips Analytical X-ray B.V. diffractometer for X-RD were available in the instrumentation lab at IIT, Roorkee. Muffle furnace (TANCO, Shivaki-T 701) with digital tempreture controller(up to $1000^{\circ} \mathrm{c}$ ), Oven (NSW INDIA) model I-43(temp up to $250^{\circ}$ c), Rotary Shaker (TANCO) with a capacity of 16 conical flasks $(250 \mathrm{ml})$. Samson Electronic balance(Model-300D) and Magnetic stirrer with hot plate were used i

\section{SYNTHESIS :-}

Six samples of bismuth(III) Iodomolybdate were prepared by mixing $0.1 \mathrm{M}$ solution of Bismuth nitrate , $0.1 \mathrm{M}$ solution of potassium iodate and $0.1 \mathrm{M}$ solution of sodium molybdate with continuous stirring in various mixing volume ratios (Table-I). The precipitates were dried at $40 \pm 1^{\circ} \mathrm{C}$. The dried products were cracked when immersed in water just after drying. The granules were charged by keeping them in with $1 \mathrm{M}$ nitric acid solution for $24 \mathrm{hrs}$ with occasional shaking and intermittently changing the acid solution. The products were washed with distilled water to free from acid and then dried at $40 \pm 1^{\circ} \mathrm{C}$ in a temperature controlled oven. The materials, so obtained were creamy white in colour and were ready to use as cation exchangers (Table- 2).

\section{Characterization:-}

Determination of ion exchange capacity for $\mathrm{Na}^{+}$ions by column method :The ion exchange capacity of all the six samples were determined (Table-3).

Synthesis of the selected sample in bulk for detailed study :- 
The sample BIM-2 which was synthesized in bulk, was characterized on the basis of different parameters as given below:-

\section{Determination of Ion exchange capacity for different metal ions by column method:-}

Ion exchange capacity of BIM-2 was determined in meq/g (dry exchanger) for various metal ions are reported in (Table-4).

\section{Determination of Ion exchange capacity by $\mathrm{P}^{\mathrm{H}}$ titration method:-}

$\mathrm{p}^{\mathrm{H}}$ titration method is also used for the determination of ion exchange capacity of the selected sample(Table-5). The $\mathrm{P}^{\mathrm{H}}$ of the different solutions were recorded after equilibrium and plotted against the strength of the $\mathrm{OH}^{-}$ions (fig-1).

\section{Thermal stability :-}

Loss in weight of the seven samples were recorded(Table-7). Ion exchange capacities of all the samples after heating were also determined(Table-6). The graph was plotted between temperature and Ion exchange capacity(fig-2). The graph was also plotted between temperature andt weight loss (fig.-3)

\section{Chemical Stability : -}

After $24 \mathrm{hrs}$ loss in weight and change in colour of the samples were recorded (Table - 8). After treating the material, ion exchange capacities of all the samples were determined by column method in (Table9).

\section{Distribution Studies :-}

The distribution coefficient is inversely proportional to the concentration of the solution. Distribution studies were carried out for several metal ions by batch $\operatorname{process}^{43}($ Table-10). Selectivity coefficient (Kd) values were calculated using the following expression :-

Where,

$$
\mathrm{Kd}=\frac{[\mathrm{I}-\mathrm{F}]}{\mathrm{F}} \cdot \frac{\mathrm{V}}{\mathrm{M}}
$$

I = Initial amount of the ions in the solution phase,

$\mathrm{F}=$ Final amount of the ions in the solution phase,

$\mathrm{V}=$ Volume of the solution $(\mathrm{ml})$,

$\mathrm{W}=$ Weight of the exchanger $(\mathrm{g})$.

The IR absorption spectrum was recorded in (Fig. 4).

\section{X-Ray diffraction :-}

The X-ray diffraction pattern of the exchanger was also obtained from Instrumentation Centre, IIT Roorkee where Philips analytical X-ray B.V. Diffractometer was available. The diffraction pattern is exhibited by (fig. 5) which shows a number of peaks.

\section{Thermogravimetric Analycis Curve:-}

The TGA curve of Bismuth(III) Iodomolybdate was studied (fig. 6). The thermogram of Bismuth(III) Iodomolybdate exhibited weight loss up to $200^{\circ} \mathrm{C}$, which is due to the removal of water molecules.

\section{Results \& Discussion :}

Creamy white precipitate of Bismuth(III) Iodomolybdate were obtained (Table- 1, Table-2). In all the six samples, ion exchange capacity of sample no. 2 was found to be maximum (Table-3). Ion exchange capacity of the selected sample with some monovalent and bivalent cations have been determined (Table-4). The maximum ion exchange capacity was found to be $1.14 \mathrm{meq} / \mathrm{g}$ for $\mathrm{Na}^{+}$ions. The ion exchange capacities were found in the order as given below (Table-4):-
$\mathrm{Na}^{+}$
$>\quad \mathrm{K}^{+}$
$\mathrm{Ca}^{2+}>$
[Alkali metal]
$\mathrm{Mg}^{2+}$
$>$
$\mathrm{Ba}^{2+}$
[Alkaline metal]

The exchanger remained solid up to $300^{\circ} \mathrm{C}$ (Table- 6) and (Table-7). The material was found to be fairly stable in $\mathrm{M} \mathrm{H}_{2} \mathrm{SO}_{4}, \mathrm{M} \mathrm{HNO}_{3}$ and dissolve in $2 \mathrm{M} \mathrm{HCl}$. It was found slightly stable in $\mathrm{M} \mathrm{KOH}, \mathrm{M} \mathrm{NaOH}$ and $\mathrm{M} \mathrm{NaNO}_{3}$ and completely soluble in $2 \mathrm{M} \mathrm{NaOH}$ and $2 \mathrm{M} \mathrm{KOH}$ solution. Effect of chemicals was determined in terms of loss in weight and loss in IEC (Table-8, Table-9). 
The $\mathrm{P}^{\mathrm{H}}$ titration curve (fig-1) showed that the ion exchange material releases $\mathrm{H}^{+}$ions easily on addition of $\mathrm{NaCl}$ solution to the system. The ion exchange capacity calculated from the titration curve at $\mathrm{P}^{\mathrm{H}} 7.0$ was 1.2 $\mathrm{meq} / \mathrm{g}$, which is in close agreement to those obtained by the column method.

The thermogram (fig-6) of Bismuth(III) Iodomolybdate exhibited weight loss up to $200^{\circ} \mathrm{C}$, which is due to the removal of water molecules. Further a gradual loss of weight of Ion exchanger was attributed to the condensation of $\mathrm{IO}_{3}{ }^{-}$group in to $\mathrm{I}_{2} \mathrm{O}_{5}$. The effect of heating on I.E.C. of Bismuth(III) Iodomolybdate from $50^{\circ} \mathrm{C}$ to $350^{\circ} \mathrm{C}$ showed that the I.E.C. decreases with increase in temperature. In order to further characterize the materials its FTIR study (fig - 4) was done. The strong and broad absorption band ${ }^{46}$ in the region $3600-3000 \mathrm{~cm}^{-}$ ${ }^{1}$ may be assigned to interstitial water molecules and free-OH groups. Another sharp peak between $1600-1650$ $\mathrm{cm}^{-1}$ may be due to the $\mathrm{H}-\mathrm{OH}$ bending motion which produces a medium band in this region.

The X-ray diffraction pattern of Bismuth(III) Iodomolybdate exhibits quite weak peaks, indicating its amorphous form (fig - 5).

Distribution studies of the studied metal ions revealed that Bismuth(III) Iodomolybdate is selective for $\mathrm{Ni}^{+2}$ ions.

Table-1

Synthesis of Bismuth(III) Iodomolybdate

\begin{tabular}{|c|c|c|c|c|c|c|c|c|}
\hline \multirow{2}{*}{$\begin{array}{l}\text { Sl. } \\
\text { No. }\end{array}$} & \multirow{2}{*}{$\begin{array}{l}\text { Sample } \\
\text { Number }\end{array}$} & \multicolumn{3}{|c|}{ Molarity of Solution } & \multirow{2}{*}{$\begin{array}{c}\text { Mixing } \\
\text { Volume } \\
\text { Ratio } \\
\text { (BN: PI: } \\
\text { SM) }\end{array}$} & \multirow[t]{2}{*}{$\overline{\text { pH }}$} & \multirow{2}{*}{$\begin{array}{l}\text { Precipitates } \\
\text { Drying temp. } \\
\left({ }^{\circ} \mathbf{C}\right)\end{array}$} & \multirow{2}{*}{$\begin{array}{l}\text { Colour of } \\
\text { precipitates }\end{array}$} \\
\hline & & BN & PI & SM & & & & \\
\hline 1. & BIM-1 & $0.1 \mathrm{M}$ & $0.1 \mathrm{M}$ & $0.1 \mathrm{M}$ & 1:1:1 & 1 & 40 & White \\
\hline 2. & BIM-2 & $0.1 \mathrm{M}$ & $0.1 \mathrm{M}$ & $0.1 \mathrm{M}$ & $1: 2: 2$ & 1 & 40 & White \\
\hline 3. & BIM-3 & $0.1 \mathrm{M}$ & $0.1 \mathrm{M}$ & $0.1 \mathrm{M}$ & $2: 1: 1$ & 1 & 40 & White \\
\hline 4. & BIM-4 & $0.1 \mathrm{M}$ & $0.1 \mathrm{M}$ & $0.1 \mathrm{M}$ & $2: 2: 1$ & 1 & 40 & White \\
\hline 5. & BIM-5 & $0.1 \mathrm{M}$ & $0.1 \mathrm{M}$ & $0.1 \mathrm{M}$ & $3: 1: 1$ & 1 & 40 & White \\
\hline 6. & BIM-6 & $0.1 \mathrm{M}$ & $0.1 \mathrm{M}$ & $0.1 \mathrm{M}$ & $3: 2: 2$ & 1 & 40 & White \\
\hline
\end{tabular}

$\mathrm{BN}=\mathrm{Bismuth}$ nitrate, $\mathrm{PI}=$ Potassium iodate, $\mathrm{SM}=$ Sodium Molybdate. Mixing volume ratio $1=40 \mathrm{ml}, 2=80$ $\mathrm{ml}, 3=120 \mathrm{ml}$, BIM=Bismuth(III) Iodomolybdate

Table-2

Generation of Bismuth(III) Iodomolybdate

\begin{tabular}{|l|c|c|c|c|}
\hline $\begin{array}{c}\text { Sl. } \\
\text { No. }\end{array}$ & $\begin{array}{c}\text { Sample } \\
\text { Number }\end{array}$ & $\begin{array}{c}\text { Appearance of Beads }{ }^{\mathbf{1}} \text { before } \\
\text { generation }\end{array}$ & $\begin{array}{c}\text { Appearance of Beads } \\
\text { after generation }\end{array}$ & $\begin{array}{c}\text { Drying temp. } \\
\left({ }^{\circ} \mathbf{C}\right)\end{array}$ \\
\hline 1. & BIM-1 & White & Creamy white & 40 \\
\hline 2. & BIM-2 & White & Creamy white & 40 \\
\hline 3. & BIM-3 & White & Creamy white & 40 \\
\hline 4. & BIM-4 & White & Creamy White & 40 \\
\hline 5. & BIM-5 & White & Creamy white & 40 \\
\hline 6. & BIM-6 & White & & 40 \\
\hline
\end{tabular}

1. Beads are obtained from the precipitates (Refer table - 1)

Table- 3

Ion exchange capacity values of Bismuth(III) Iodomolybdate samples

\begin{tabular}{|c|c|c|c|c|}
\hline SI.No. & Sample Number & Salt Solution & $\begin{array}{c}\text { Molarity of Salt } \\
\text { Solution }\end{array}$ & I.E.C. for Na $(\mathbf{m e q} / \mathbf{g})$ \\
\hline 1. & BIM-1 & $\mathrm{NaNO}_{3}$ & $1 \mathrm{M}$ & 0.8 \\
\hline 2. & BIM-2 & $\mathrm{NaNO}_{3}$ & $1 \mathrm{M}$ & 1.2 \\
\hline 3. & BIM-3 & $\mathrm{NaNO}_{3}$ & $1 \mathrm{M}$ & 0.84 \\
\hline 4. & BIM-4 & $\mathrm{NaNO}_{3}$ & $1 \mathrm{M}$ & 0.7 \\
\hline 5. & BIM-5 & $\mathrm{NaNO}_{3}$ & $1 \mathrm{M}$ & 0.88 \\
\hline 6. & BIM-6 & $\mathrm{NaNO}_{3}$ & 0.24 \\
\hline
\end{tabular}

$\mathrm{M}=$ Molar $\mathrm{NaNO}_{3}$ solution in water 
Table- 4

I.E.C. of Bismuth(III) Iodomolybdate of some Monovalent and Bivalent Cations.

\begin{tabular}{|c|c|c|c|c|c|}
\hline $\begin{array}{c}\text { Serial } \\
\text { Number }\end{array}$ & Salt Solution & Metal ion & Molarity & $\begin{array}{c}\text { Ion Exchange } \\
\text { capacity } \\
\text { [meq/g] } \\
\end{array}$ & $\begin{array}{c}\text { Hydrated ionic } \\
\text { Radii } \\
{\left[\mathbf{A}^{\circ}\right]} \\
\end{array}$ \\
\hline 1. & Sodium Chloride & $\mathrm{Na}^{+}$ & $1 \mathrm{M}$ & 1.14 & 7.90 \\
\hline 2. & Potassium Chloride & $\mathrm{K}^{+}$ & $1 \mathrm{M}$ & 0.54 & 5.30 \\
\hline 3. & Magnisium Chloride & $\mathrm{Mg}^{2+}$ & $1 \mathrm{M}$ & 1.12 & 10.80 \\
\hline 4. & Calcium Chloride & $\mathrm{Ca}^{2+}$ & $1 \mathrm{M}$ & 0.78 & 9.60 \\
\hline 5. & Barium Chloride & $\mathrm{Ba}^{2+}$ & $1 \mathrm{M}$ & 0.56 & 8.80 \\
\hline
\end{tabular}

Table- 5

$\underline{\mathbf{P}^{\mathrm{H}} \text { titration values of Bismuth(III) Iodomolybdate }}$

\begin{tabular}{|c|c|c|c|c|}
\hline \multirow{2}{*}{$\begin{array}{c}\text { Sl. } \\
\text { No. }\end{array}$} & \multirow{2}{*}{ Sample Number } & \multicolumn{2}{|c|}{ Mixing Volume Ratio } & \multirow[t]{2}{*}{$\mathbf{P}^{\mathrm{H}}$ Value } \\
\hline & & NaCl Solution & NaOH Solution & \\
\hline 1. & BIM-2 & 50 & 00 & 2.73 \\
\hline 2. & BIM-2 & 45 & 05 & 6.63 \\
\hline 3. & BIM-2 & 40 & 10 & 8.19 \\
\hline 4. & BIM-2 & 35 & 15 & 9.28 \\
\hline 5. & BIM-2 & 30 & 20 & 9.61 \\
\hline 6. & BIM-2 & 25 & 25 & 9.94 \\
\hline 7. & BIM-2 & 20 & 30 & 9.99 \\
\hline 8. & BIM-2 & 15 & 35 & 10.75 \\
\hline 9. & BIM-2 & 10 & 40 & 11.61 \\
\hline 10. & BIM-2 & 05 & 45 & 11.65 \\
\hline 11. & BIM-2 & 00 & 50 & 12.02 \\
\hline
\end{tabular}

Table- 6

Heating Effect Study on IEC of Bismuth(III) Iodomolybdate

\begin{tabular}{|l|c|c|c|c|c|c|c|}
\hline $\begin{array}{c}\text { Sl. } \\
\text { No. }\end{array}$ & $\begin{array}{c}\text { IEC Before } \\
\text { Heating }\end{array}$ & $\begin{array}{c}\text { Heating } \\
\text { Temp. }\left({ }^{\circ} \mathbf{C}\right)\end{array}$ & $\begin{array}{c}\text { Heating } \\
\text { Time } \\
(\text { Hour })\end{array}$ & $\begin{array}{c}\text { IEC after } \\
\text { Heating }\end{array}$ & $\begin{array}{c}\text { Loss in } \\
\text { IEC }\end{array}$ & $\begin{array}{c}\text { Physical } \\
\text { State }\end{array}$ \\
\hline 1. & 1.2 & 50 & 1 & 1.00 & 0.20 & Creamy white & Solid \\
\hline 2. & 1.2 & 100 & 1 & 0.53 & 0.67 & Creamy white & Solid \\
\hline 3. & 1.2 & 150 & 1 & 0.42 & 0.78 & Creamy white & Solid \\
\hline 4. & 1.2 & 200 & 1 & 0.30 & 0.90 & Creamy white & Solid \\
\hline 5. & 1.2 & 250 & 1 & 0.17 & 1.03 & Pinkish white & Solid \\
\hline 6. & 1.2 & 300 & 1 & 0.15 & 1.05 & Shine brown & Liquid \\
\hline 7. & 1.2 & 350 & 1 & 0.13 & 1.07 & Shine brown \\
\hline
\end{tabular}

IEC = Ion exchange capacity for $\mathrm{Na}^{+}\left(\mathrm{NaNO}_{3}\right.$ solution $)$

Table- 7

Heating Effect Study on Weight of Bismuth(III) Iodomolybdate

\begin{tabular}{|c|c|c|c|c|c|c|c|}
\hline $\begin{array}{c}\text { Sl. } \\
\text { No. }\end{array}$ & $\begin{array}{c}\text { Weight of Ion } \\
\text { Exchanger Before } \\
\text { Heating (g) }\end{array}$ & $\begin{array}{c}\text { Heating } \\
\text { Temp. }\left({ }^{\circ} \mathrm{C}\right)\end{array}$ & $\begin{array}{c}\text { Heating } \\
\text { Time } \\
\text { (Hour) }\end{array}$ & $\begin{array}{c}\text { Weight of Ion } \\
\text { Exchanger after } \\
\text { Heating (g) }\end{array}$ & $\begin{array}{l}\text { Weight } \\
\text { Loss (g) }\end{array}$ & $\begin{array}{c}\% \text { Weight } \\
\text { Loss }\end{array}$ & $\begin{array}{c}\text { Physical } \\
\text { State }\end{array}$ \\
\hline 1. & 0.50 & 50 & 1 & 0.50 & 0.00 & 0 & Solid \\
\hline 2. & 0.50 & 100 & 1 & 0.49 & 0.01 & 1 & Solid \\
\hline 3. & 0.50 & 150 & 1 & 0.48 & 0.02 & 2 & Solid \\
\hline 4. & 0.50 & 200 & 1 & 0.47 & 0.03 & 3 & Solid \\
\hline 5. & 0.50 & 250 & 1 & 0.47 & 0.03 & 3 & Solid \\
\hline 6. & 0.50 & 300 & 1 & 0.46 & 0.04 & 4 & Solid \\
\hline 7. & 0.50 & 350 & 1 & 0.45 & 0.05 & 5 & Liquid \\
\hline
\end{tabular}




\section{Table-8}

Chemical Stability of Bismuth(III) Iodomolybdate

\begin{tabular}{|c|c|c|c|c|c|}
\hline Sl. Number & Solution & Molarity & Colour & $\mathrm{W}_{\mathrm{I}}(\mathrm{g})$ & $W_{F}(g)$ \\
\hline \multirow[t]{4}{*}{1} & \multirow[t]{4}{*}{$\mathrm{H}_{2} \mathrm{SO}_{4}$} & 0.1 & Creamy white & 0.25 & 0.17 \\
\hline & & 1.0 & Creamy white & 0.25 & 0.16 \\
\hline & & 0.2 & Creamy white & 0.25 & 0.18 \\
\hline & & 2.0 & Creamy white & 0.25 & 0.12 \\
\hline \multirow[t]{4}{*}{2} & \multirow[t]{4}{*}{$\mathrm{HNO}_{3}$} & 0.1 & Creamy white & 0.25 & 0.19 \\
\hline & & 1.0 & Creamy white & 0.25 & 0.14 \\
\hline & & 0.2 & Creamy white & 0.25 & 0.19 \\
\hline & & 2.0 & Creamy white & 0.25 & 0.08 \\
\hline \multirow[t]{3}{*}{3} & \multirow[t]{3}{*}{$\mathrm{HCl}$} & 0.1 & Creamy white & 0.25 & 0.16 \\
\hline & & 1.0 & C.D. & 0.25 & C.D. \\
\hline & & 0.2 & Creamy white & 0.25 & 0.07 \\
\hline \multirow[t]{2}{*}{4} & \multirow[t]{2}{*}{$\mathrm{NaOH}$} & 1.0 & Yellow & 0.25 & 0.06 \\
\hline & & 0.2 & C.D. & 0.25 & C.D. \\
\hline \multirow[t]{2}{*}{5} & \multirow[t]{2}{*}{$\mathrm{KOH}$} & 1.0 & Yellow & 0.25 & 0.08 \\
\hline & & 0.2 & C.D. & 0.25 & C.D. \\
\hline \multirow[t]{3}{*}{6} & \multirow[t]{3}{*}{$\mathrm{NaNO}_{3}$} & 1.0 & Light lemon & 0.25 & 0.17 \\
\hline & & 0.2 & Light lemon & 0.25 & 0.20 \\
\hline & & 2.0 & Light lemon & 0.25 & 0.09 \\
\hline
\end{tabular}

$W_{I}=$ Initial weight of exchanger before dipping in solution $W_{F}=$ Final weight of exchanger after dipping in solution C.D.= Completely dissolved exchanger in solution

Table- 9

Ion exchange capacity of Bismuth(III) Iodomolybdate against Chemicals

\begin{tabular}{|c|c|c|c|c|}
\hline Sl.No. & Sample Number & Solution & Molarity & $\overline{\text { Ion exchange capacity }}$ \\
\hline 1. & BIM-2 & $\mathrm{H}_{2} \mathrm{SO}_{4}$ & 0.1 & 0.4 \\
\hline 2. & BIM-2 & $\mathrm{H}_{2} \mathrm{SO}_{4}$ & 1.0 & 0.3 \\
\hline 3. & BIM-2 & $\mathrm{H}_{2} \mathrm{SO}_{4}$ & 0.2 & 0.8 \\
\hline 4. & BIM-2 & $\mathrm{H}_{2} \mathrm{SO}_{4}$ & 2.0 & 0.9 \\
\hline 5. & BIM-2 & $\mathrm{HNO}_{3}$ & 0.1 & 0.9 \\
\hline 6. & BIM-2 & $\mathrm{HNO}_{3}$ & 0.2 & 0.7 \\
\hline 7. & BIM-2 & $\mathrm{HNO}_{3}$ & 2.0 & 0.8 \\
\hline 8. & BIM-2 & $\mathrm{HNO}_{3}$ & 0.1 & - \\
\hline 9. & BIM-2 & $\mathrm{HCl}$ & 1.0 & 0.7 \\
\hline 10. & BIM-2 & $\mathrm{HCl}$ & 0.2 & - \\
\hline 11. & BIM-2 & $\mathrm{HCl}$ & 1.0 & - \\
\hline 12. & BIM-2 & $\mathrm{NaOH}$ & 0.2 & - \\
\hline 13. & BIM-2 & $\mathrm{NaOH}$ & 1.0 & - \\
\hline 14. & BIM-2 & $\mathrm{KOH}$ & 0.2 & - \\
\hline 15. & BIM-2 & $\mathrm{KOH}$ & 1.0 & - \\
\hline 16. & BIM-2 & $\mathrm{NaNO}_{3}$ & 0.2 & 1.2 \\
\hline 17. & BIM-2 & $\mathrm{NaNO}_{3}$ & 2.0 & 1.1 \\
\hline 18. & BIM-2 & $\mathrm{NaNO}_{3}$ & 2.0 & - \\
\hline
\end{tabular}

Table- 10

Distribution Coefficient (Kd) values for metal ions

\begin{tabular}{|c|c|c|c|}
\hline Sl. Number & Cations & Taken as & Kd (ml/g ) \\
\hline 1 & $\mathrm{Mn}^{2+}$ & Sulphate & 62.61 \\
\hline 2 & $\mathrm{Zn}^{2+}$ & Sulphate & 12.73 \\
\hline 3 & $\mathrm{Mg}^{2+}$ & Chloride & 85 \\
\hline 4 & $\mathrm{Ni}^{2+}$ & Sulphate & 120 \\
\hline 5 & $\mathrm{Cd}^{2+}$ & Nitrate & 31.58 \\
\hline 6 & $\mathrm{Co}^{2+}$ & Sulphate & 28.57 \\
\hline 7 & $\mathrm{~Pb}^{2+}$ & Nitrate & 45.60 \\
\hline
\end{tabular}


Fig. -1

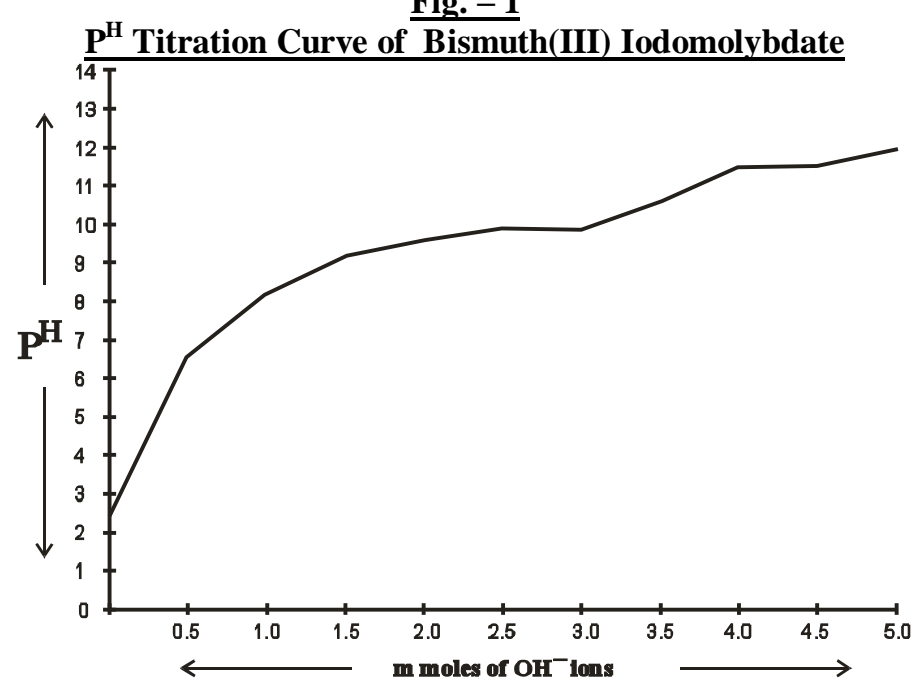

Fig. -2

Thermal stability curve between temperature and weight loss of Bismuth(III) Iodomolybdate

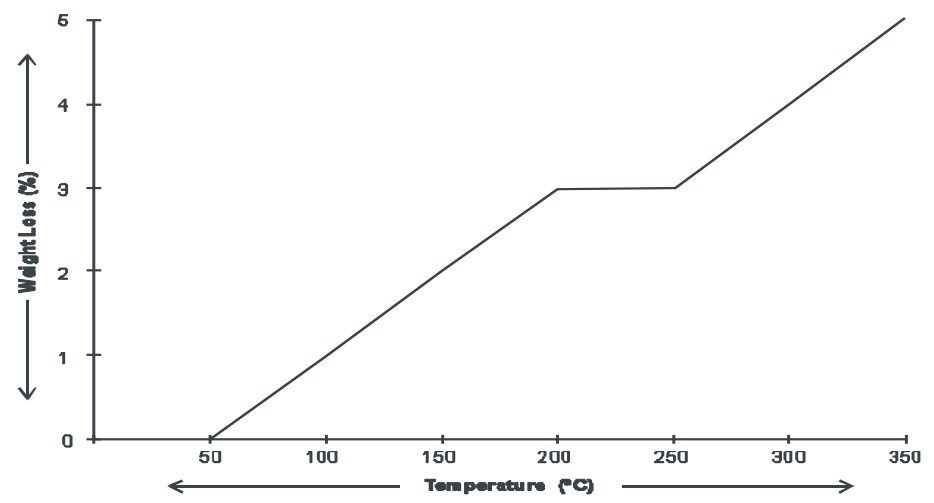

Fig. -3

Thermal stability curve between temperature and ion exchange capacity of Bismuth(III) Iodomolybdate

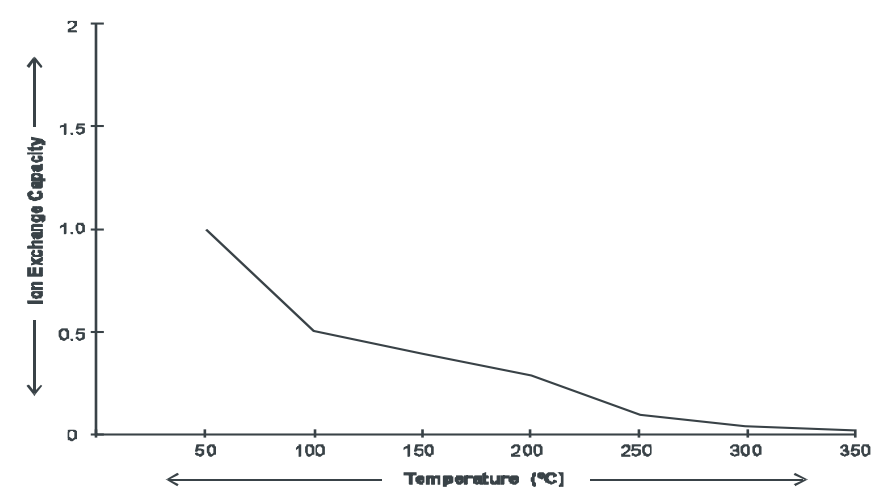


Fig. -4

IR of Bismuth(III) Iodomolybdate

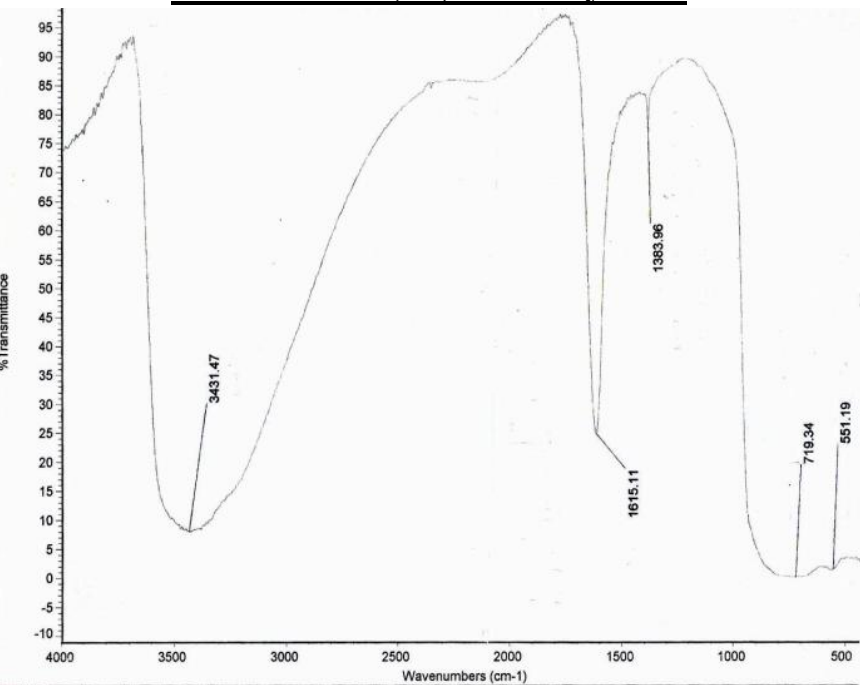

Fig. -5

$\underline{\text { XRD pattern of Bismuth(III) iodomolybdate }}$

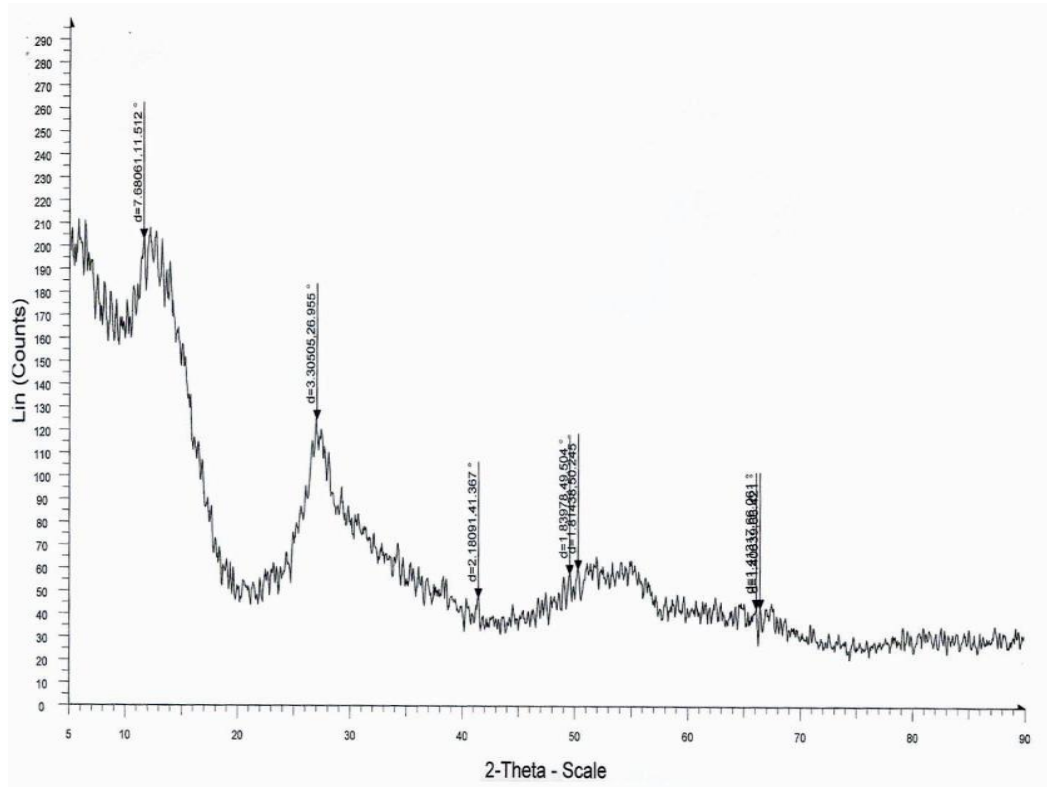

Fig. -6

TGA of Bismuth(III) Iodomolvbdate

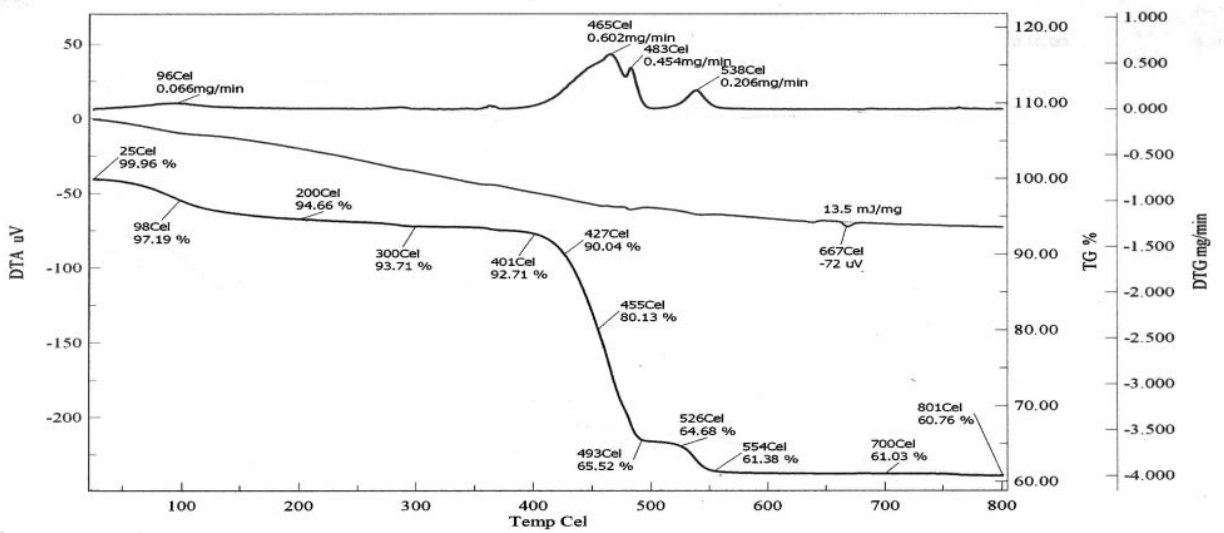


Fig. -7

Specificity of Bismuth(III) Iodomolybdate of $\mathrm{Ni}^{++}$and $\mathrm{Cd}^{++}$

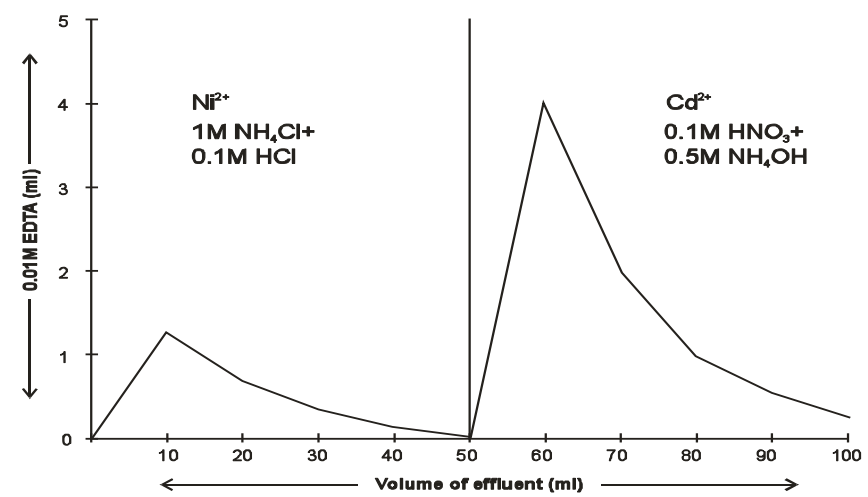

Fig. -8

Specificity of Bismuth(III) Iodomolybdate of $\mathrm{Mn}^{++}$and $\mathrm{Ni}^{++}$

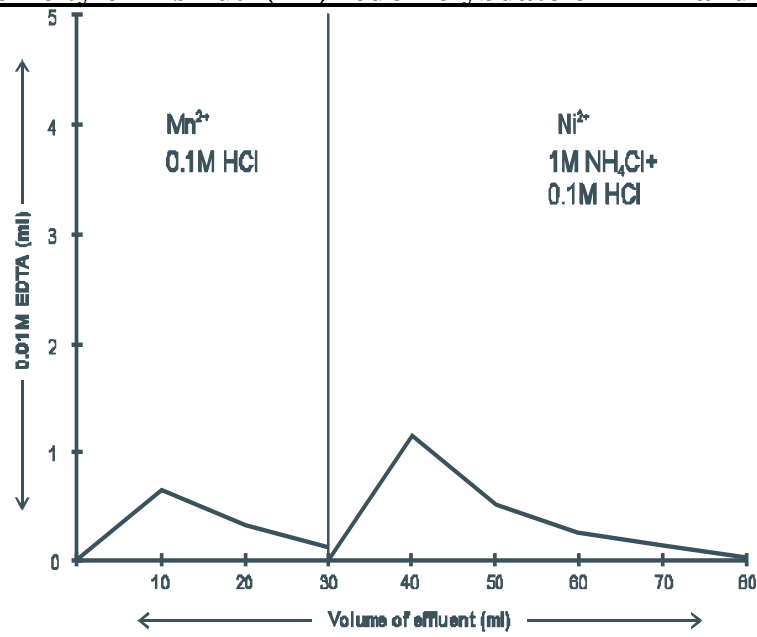

Fig. - 9

Specificity of Bismuth(III) Iodomolybdate of $\mathrm{Zn}^{++}$and $\mathrm{Ni}^{++}$

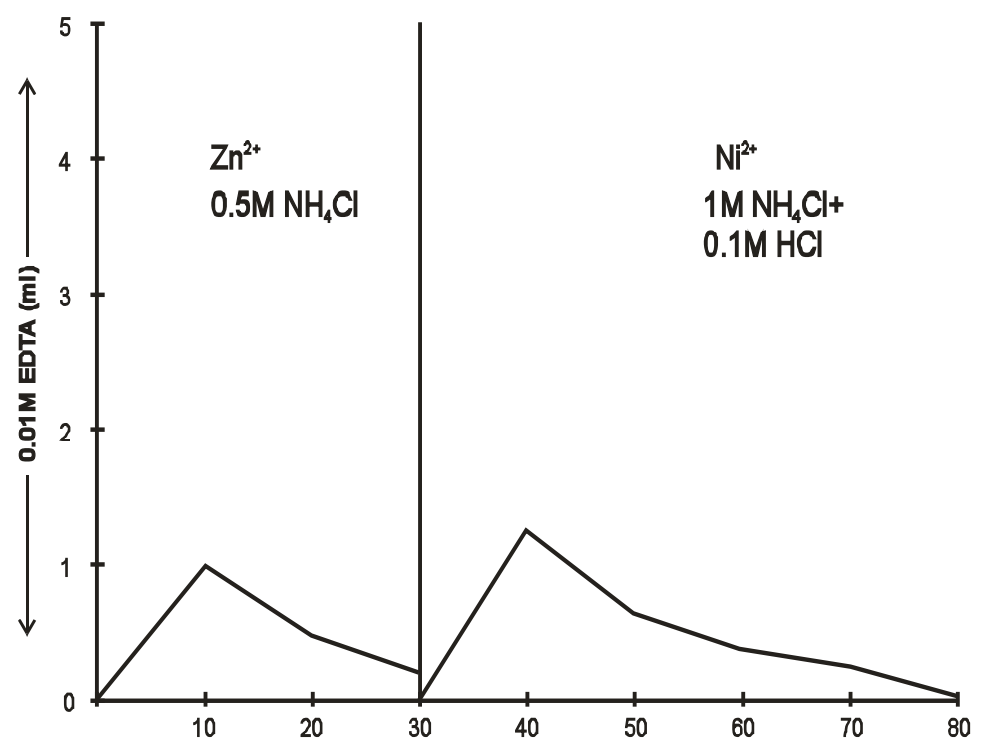


Fig. - 10

Specificity of Bismuth(III) Iodomolybdate of $\mathrm{Pb}^{++}$and $\mathrm{Ni}^{++}$

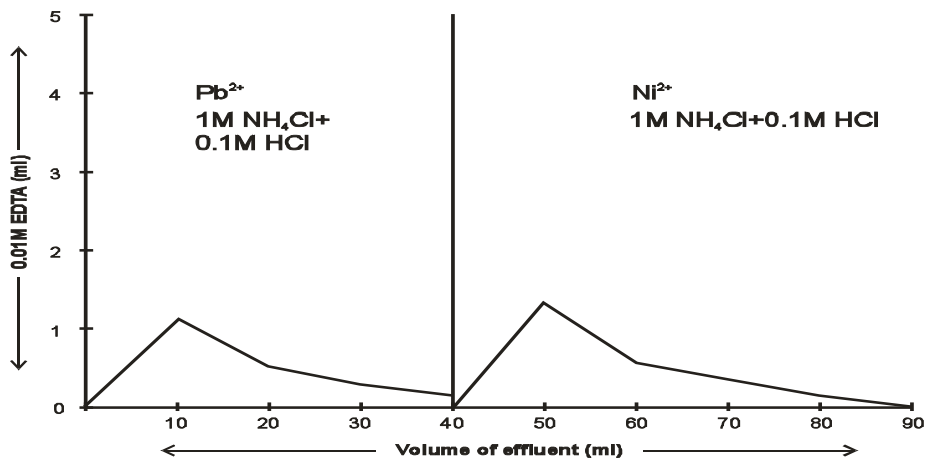

References

[1]. Pohl chris; recent development in ion exchange columns for inorganic ions and low molecular weight molecules, 22 LC GC LC column technology supplement June 2004.

[2]. Samuelson O. ; Ion Exchangers in Analytical Chemistry, John Wiley and Sons \& Inc., New York, 1954.

[3]. Singh, D.K. Srivastava Bhavana \& Yadav Pushpa; J. Indian chem. Soc. 80, 866, 2003.

[4]. Ismail A.A., Mohamed R.M., Ibrahim I. A.,Kini G., and Koopman B.; Colloids and Surfaces A : Physicochemical and Engineering Aspects, 366(1-3), 80-87, 20 August 2010.

[5]. Clearfield A. ; Inorganic ion exchange materials ( CRC Press, Florida, USA) 1982.

[6]. Qureshi M. \& Varshney K. G. ; Inorganic ion exchangers in chemical analysis (C R C Press, Florida, USA) 1991.

[7]. Sharma S. D., Mishra S. \& Gupta A. ; Indian J. Chem, 33 A (1994 ) 696.

[8]. Gupta A. P. \& Renuka ; Indian J. Chem , 36 (1997 ) 1073.

[9]. Turel Z. R. \& Narkhade S. S. ; J. Indian Chem soc, 75 (1998) 1772. 